\title{
Robust immersion and invariance adaptive synchronization control with disturbance observer for maglev module with output constraint ${ }^{\text {th }}$
}

\author{
Jiaqi Liu, Xiangbin Liu* and Min Zhang \\ School of Electronic Information Engineering, Beijing Jiaotong University, Beijing \\ 100044, People's Republic of China
}

\begin{abstract}
Magnetic levitation module is the key component of maglev train that generates the electromagnet force to bear the weight of the vehicle cabin. In this paper, the mathematical model of the levitation module is built as a two-input and two-output coupled system whose state variables include the vertical linear motion and the rotational motion around the mass center, which fully considers the mechanical coupling and force interference. To alleviate the variation of the module, a framework of robust immersion and invariance (I\&I) adaptive synchronization control with disturbance observer is presented for the levitation module. To deal with the bad effects of parametric uncertainties and external disturbances on the system performance, especially on system outputs, we introduce Barrier Lyapunov Function (BLF) into controller to keep the outputs in the prescribed constraints. The robust I\&I adaptation law is used to shape the converging procedure of the estimate errors of the parametric uncertainties. An extended disturbance observer is developed to recover the mismatched disturbances of the maglev module simultaneously. The stability analysis shows that all the signals in the closed-loop system are bounded and output constraints are not violated as well. The effectiveness of the proposed scheme is verified by a
\end{abstract}

*Corresponding author:Xiangbin Liu, xbliu@bjtu.edu.cn

Preprint submitted to Journal of $E^{A} T_{E} X$ Templates

July 20, 2021 
numerical simulations at last.

Keywords: Levitation module, Mechanical coupling, Rotational

dynamics, Output constraints, Disturbance observer, Immersion and invariance

\section{Introduction}

Maglev train technology has been widely investigated in the past few decades due to its advantages such as lower noise, less maintenance cost, less exhaust fumes emission and ride comfort 11. The worldwide interest on the research of

5 maglev train leads to success establishment of several commercial maglev routes and test routes in China, Japan, and Korea [2, 3].

For EMS type urban maglev vehicle, early research usually simplified the vehicle as a single point [4] or combined multi-points model [5, 6], which is hard to reflect the actually dynamic characteristics of the vehicle. Therefore, it is of importance to improve the model precision of the vehicle. In fact, there exist 4-5 independent bogies working simultaneously to bear the cabin weight through air springs. Each bogie comprises two levitation modules and the overall weight of cabin is distributed to every levitation modules. These levitation modules are mechanically "isolated" from each other proved by the experiment undertaking on the Beijing $S 1$ maglev vehicle 7 . Consequently, the controller for every levitation module is designed separately and the influences among levitation modules are neglected [8].

The levitation module of maglev train consists of two integrated electromagnet units with four coils which are divided into two groups along the direction of travelling. The two coils in each group are connected in series and the electric current passing through them is controlled by corresponding levitation controller. The existing control strategy controls the two units of the levitation module separately without communications between the two controllers. It ignores the mechanical coupling between the front and the rear levitation units 25 since the four coils are assembled on the same pair of magnetic poles, which 
would cause large force variation as the two controllers are not synchronized to manipulate levitation. Motivated by a synchronization control scheme proposed for a dual-linear-motor-driven (DLMD) gantry to deal with the mechanical coupling problem in [9, 10, the nonlinear mathematical model of the levitation module is built as two-input and two-output coupled system, whose state variables include vertical linear motion and the rotational motion around the mass center in this paper.

Since EMS maglev train is an open-loop unstable system and its maglev models are highly nonlinear dynamics with many kinds of uncertainties and subjected to external disturbances due to track irregularity and air pressure change as well, it puts strict requirements on controller design to avoid cabin large vibration as the train operates. The large vibration not only deteriorates passenger ride comfort, but also may cause collision between the cabin and rail track. To alleviate the variation, it is reasonable to predefine constraints 40 on the outputs of the two units. There exists some effective way, especially Barrier Lyapunov Function, to deal with output constraint 11, 12. In [1], the symmetric output constraint is achieved by utilizing Barrier Lyapunov Function for the single-input single-output (SISO) nonlinear systems in strict feedback form. 12 employs the asymmetric time-varying Barrier Lyapunov Function 45 for output tracking problems of a class of multi-input multi-output (MIMO) nonlinear systems to guarantee desired tracking performance.

On the other hand, various kinds of uncertainties and external disturbances of maglev module have negative impacts on control performance. The rejection or attenuation of these uncertain terms of maglev systems in control law design is shown in mismatched form, for example, the channels of disturbance from track entering into maglev vehicle system is different from the control input$\mathrm{s}$ [13, 14. To remove the negative impact of mismatched disturbances on a system, several kinds of disturbance observers (DO) are proposed to improve robustness for single-input and single-output nonlinear systems [15], and multiinput multi-output systems [16. A disturbance observer based on sliding mode 
control is developed for the system with mismatched disturbances using a new sliding surface that includes the disturbance estimation in [18]. [19] proposes a novel disturbance observer based robust control to counteract the mismatched disturbance for an uncertain system from the output channels. However, the disturbance in [18, 19] are vanishing type in steady state. The requirement always cannot be satisfied in real applications such as the disturbance of maglev train exists in the whole operation because the irregularity along the track is a function of displacement whose derivatives with respect to displacement are bound but do not converge to zero. In [20], the mismatched disturbances shown in the form of time-varying functions can be estimated by an extend disturbance observer, which is applicable to deal with the disturbances in maglev system in this paper.

It is worth pointing out that load mass of maglev train may also change greatly, which may make the system performance worse and even dangerous to maglev train operation [21, 22, 23]. Therefore, it is necessary to design adaptation law to estimate such unknown parameters (load mass and/ or other parametric uncertainties) on-line for compensation.

In [25, a novel design method called immersion and invariance (I\&I) mani75 fold is proposed, which is suitable for adaptive algorithm design. The key merit of I\&I method is to avoid the complexity of constructing Lyapunov functions in the adaptation control design. It obtains great attention since introduction of a suitable tuning function not only provides freedom to shape the convergence dynamics but also renders the estimated errors to exponentially converge to a residual set, which is different from the traditional adaptive algorithm based on the certainty equivalence principle [26].

In this paper, a synchronization controller for the output-constrained uncertain maglev module subjected to mismatched disturbances is proposed. Since the structure of the levitation module would lead to large differences of the air 85 gap between the front and the rear levitation units, levitation module is modelled as two-input and two-output coupled system. Barrier Lyapunov Functions is used to synthesize the base-line controller to prevent the outputs of levita- 
tion module from large variation and a robust I\&I adaptation law inspired by our previous work 27] is adopted to recover the unknown parameters in levitation module, and an extended disturbance observer is constructed to handle the mismatched disturbance in the levitation module simultaneously to obtain satisfying control performance of the closed-loop system.

The rest of the paper is organized as follows. Section II describes the modeling of the levitation module subjected to mismatched disturbance and output constraint. Section III develops a robust adaptive synchronization control law to guarantee the satisfying performance of system, in which the unknown parameters are recovered via I\&I adaptation method and the mismatched disturbance is estimated by a extended disturbance observer simultaneously. The stability of closed-loop maglev module with output constraint is analyzed in Section IV.

100 Section $V$ gives the numerical simulations to verify effectiveness of the proposed controller. The conclusion is given in Section VI.

\section{Problem formulation}

\subsection{Modeling of the levitation module}

Inspired by the modeling of the DLMD gantry in [9, 10, the model of levitation module are built in this section.

The structure of the levitation module is illustrated in Fig. 1] and the moving part mainly consists of the front and the rear levitation units with link along the Y-axis. $m$ and $J$ represent the mass and rotational inertia around the geometric center $O$ of the levitation module, respectively. $g$ is the gravity acceleration. $N_{1}(t), N_{2}(t)$ denote the weight of the carriage (including load or passengers) applied equivalently to both ends of the bogie through the air springs. The variable $y_{o}$ indicates the displacement between the track and the mass center $O$ in the vertical direction and $y_{1}, y_{2}$ are the relative displacement from the track to the ends of levitation module in inertial space, which can be measured directly by the displacement sensors mounted on the electromagnet, respectively. $F_{1}$ and $F_{2}$ are the resultant electromagnetic forces of the front and rear levitation units. In this paper, the resultant electromagnetic forces can be located at 


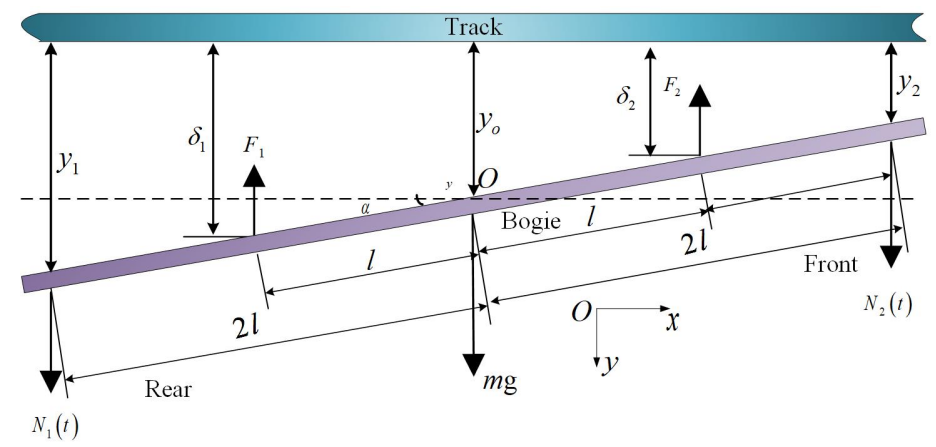

Figure 1: Schematic of the levitation module.

the middle of mass center and the end of bogie, i.e., $l_{1}=l_{2}=l_{3}=l_{4}=l$. Denote $\delta_{1}, \delta_{2}$ are the relative displacements from the action points of $F_{1}, F_{2}$ to the track, respectively. All these displacement variables are appointed to be positive when their directions are downwards. $\alpha$ is the rotational angle of the levitation module which is positive when it turns anticlockwise.

The two-leviation module are mechanically coupled, which would result in the module oscillation if the two controllers cannot manipulate corresponding levitation gap synchronically. Furthermore, large rotational angle around the mass center of the levitation module may result in collision between the electromagnets and the track due to large variation of the two levitation gaps. The following assumptions are made on building the dynamic model of the levitation module: (1) The leakage flux, the magnetic field edge effect, the magnetic resistance of iron core and track are ignored. (2) The mass density of the carriage is uniform and the mass center coincides with the geometric center of the module. (3) The value of $\alpha$ in operation is small such that $\alpha \approx \tan \alpha \approx \sin \alpha$.

The motions of the levitation module in the local coordinate system consist of the motion of the mass center along the $\mathrm{Y}$-axis and an rotation motion around the mass center. The relationships between $y_{o}, \alpha$ and $y_{1}, y_{2}$ measured by the gap sensors as

$$
y_{1}=y_{o}+2 l \alpha, \quad y_{2}=y_{o}-2 l \alpha
$$


It is easy to obtain that

$$
y_{o}=\frac{y_{1}+y_{2}}{2}, \quad \alpha=\frac{y_{1}-y_{2}}{4 l}
$$

Due to changing of the load, $N_{1}(t), N_{2}(t)$ can be described as $N_{1}(t)=$ $N_{01}+\Delta N_{1}(t), N_{2}(t)=N_{02}+\Delta N_{2}(t)$, where $N_{01}, N_{02}$ denote the nominal load values and $\Delta N_{1}(t)$ and $\Delta N_{2}(t)$ the variation of force.

From Fig 1 the two action points of the module are symmetrical to the geometrical center of the module, the gap sensors are installed at the end of each unit, which indicates that the positions of the sensors and the actuators are different. As a result, the mechanical coupling between the two levitation units may deteriorate the control performance if the corresponding controller works independently. Hence, to realize synchronization of the two controllers, it is necessary to transform the variables from $y_{1}, y_{2}$ to $y_{o}, \alpha$. Considering the module structure in Fig.1, its dynamics can be given as follows,

$$
\begin{gathered}
m \ddot{y}_{o}=m g+N_{01}+N_{02}-F_{1}-F_{2}+\Delta_{y} \\
J \ddot{\alpha}=2 l N_{01}-2 l N_{02}-l F_{1}+l F_{2}+\Delta_{\alpha}
\end{gathered}
$$

where $\Delta_{y}=d_{y}+\Delta N_{1}(t)+\Delta N_{2}(t), \Delta_{\alpha}=d_{\alpha}+2 l \Delta N_{1}(t)-2 l \Delta N_{2}(t), d_{y}$ and $d_{\alpha}$ denote the lumped external disturbances.

It is known that electromagnetic force $F$ is determined by $F=\frac{K i^{2}}{\delta^{2}}$, where $K$ is the coefficient related to magnetic unit, $i$ is the coil current of the electromagnet, $\delta$ is the relative displacement between the electromagnet and the track. From Fig, 1 , we have $\delta_{1}=y_{o}+\alpha l$ and $\delta_{2}=y_{o}-\alpha l$. Thus, the electromagnetic forces of the modules can be expressed as

$$
\begin{gathered}
F_{1}=\frac{K i_{1}^{2}}{\delta_{1}^{2}}=\frac{K i_{1}^{2}}{\left(y_{o}+\alpha l\right)^{2}}, \\
F_{2}=\frac{K i_{2}^{2}}{\delta_{2}^{2}}=\frac{K i_{2}^{2}}{\left(y_{o}-\alpha l\right)^{2}}
\end{gathered}
$$

where $i_{1}$ and $i_{2}$ are the module currents of the electromagnets, respectively. 
Note that (3) and (4), the dynamics of the module can be described as,

$$
\begin{gathered}
\ddot{y}_{o}=\frac{N_{01}}{m}+\frac{N_{02}}{m}+g-\frac{K i_{1}^{2}}{m\left(y_{o}+\alpha l\right)^{2}}-\frac{K i_{2}^{2}}{m\left(y_{o}-\alpha l\right)^{2}}+\frac{\Delta_{y}}{m} \\
\ddot{\alpha}=\frac{2 l N_{01}}{J}-\frac{2 l N_{02}}{J}-\frac{K l i_{2}^{2}}{J\left(y_{o}+\alpha l\right)^{2}}+\frac{K l i_{2}^{2}}{J\left(y_{o}-\alpha l\right)^{2}}+\frac{\Delta_{\alpha}}{J}
\end{gathered}
$$

Remark 1. In maglev train system, since the change of the current signal, controlled by input voltage, is much faster than that of the displacement signal, the lag effect of the current loop to input voltage can be ignored thus. Then, the displacement dynamics is directly controlled by coil current in this paper.

To facilitate the design of synchronization controller, the dynamics (5) is rewritten in the form of state $q=\left[y_{o}, \alpha\right]^{T}$ as follows,

$$
\ddot{q}=\theta_{2} v+\phi \theta_{1}+d(t)
$$

where $d(t)=\left[d_{1}(t), d_{2}(t)\right]^{T}=\left[\frac{\Delta_{y}}{m}, \frac{\Delta_{\alpha}}{J}\right]^{T}, \theta_{1}=\left[\theta_{11}, \theta_{12}, \theta_{13}, \theta_{14}, \theta_{15}\right]^{T}=\left[\frac{N_{01}}{m}, \frac{N_{02}}{m}\right.$, 165 $\left.g, \frac{N_{01}}{J}, \frac{N_{02}}{J}\right]^{T}$ and $\theta_{2}=\operatorname{diag}\left[\theta_{21}, \theta_{22}\right]=\operatorname{diag}\left[-\frac{K}{m},-\frac{K}{J}\right]$ are the unknown parameters in (5), $\phi \in \mathbb{R}^{2 \times 5}$ is the regressor matrix

$$
\phi=\left[\begin{array}{ccccc}
1 & 1 & 1 & 0 & 0 \\
0 & 0 & 0 & 2 l & -2 l
\end{array}\right],
$$

$v=\left[v_{1}, v_{2}\right]^{T}$ is the transformation of $u=\left[u_{1}, u_{2}\right]^{T}=\left[i_{1}^{2}, i_{2}^{2}\right]^{T}$ as

$$
v=\left[v_{1}, v_{2}\right]^{T}=T u, T=\left[\begin{array}{cc}
\frac{1}{\left(y_{o}+\alpha l\right)^{2}} & \frac{1}{\left(y_{o}-\alpha l\right)^{2}} \\
\frac{l}{\left(y_{o}+\alpha l\right)^{2}} & -\frac{l}{\left(y_{o}-\alpha l\right)^{2}}
\end{array}\right]
$$

To improve ride quality and avoid collision between the electromagnets and the track, $y_{o}(t)$ and $\alpha(t)$ are required to satisfy the following conditions,

$$
\begin{array}{r}
\left|y_{o}(t)\right| \leq k_{c 1}, \forall t \geq 0 \\
|\alpha(t)| \leq k_{c 2}, \forall t \geq 0
\end{array}
$$

where $k_{c 1}, k_{c 2}$ are given positive constants.

Remark 2. From Fig1, due to the output constraint, the physical quantities $\delta_{1}=y_{o}+\alpha l>0$ and $\delta_{2}=y_{o}-\alpha l>0$ during the train operation, which can avoid the singularity of the transformation matrix $T$ in $(7)$. 
The following reasonable assumptions of $(6)$ are given as follows.

175 Assumption 1. The disturbances $d(t)$ in (6) are continuous and bounded, satisfying the following conditions

$$
\left\|\frac{d^{j} d(t)}{d t^{j}}\right\| \leq \mu, \quad \text { for } \quad j=0,1,2, \ldots, p,
$$

where $\mu>0$ is an unknown constant and $p$ is an positive integer related to the position of disturbances $d(t)$ shown in system.

Assumption 2. For any $k_{c i}>0$, there exist five positive constants $\underline{Y}_{0 i}, \bar{Y}_{0 i}, A_{0 i}, Y_{1 i}$, $Y_{2 i}$ satisfying $\max \left\{\underline{Y}_{0 i}, \bar{Y}_{0 i}\right\} \leq A_{0 i}<k_{c i}, i=0,1$ such that the desired trajectory signals $y_{d}$ and $\alpha_{d}$ and their time derivatives satisfy $-\underline{Y}_{01} \leq y_{d} \leq \bar{Y}_{01}$, $\left|\dot{y}_{d}\right|<Y_{11},\left|\ddot{y}_{d}\right|<Y_{21}$ and $-\underline{Y}_{02} \leq \alpha_{d} \leq \bar{Y}_{02},\left|\dot{\alpha}_{d}\right|<Y_{12},\left|\ddot{\alpha}_{d}\right|<Y_{22}$.

Remark 3. The bounds of $d(t)$ and its time derivatives in Assumption 1 are used to determine the ultimate bounds of the signals in the closed-loop system. $\mu$ is only used to analysis and is not needed to be known. Assumption 2 is reasonable requirement on the desired trajectory signals $y_{d}$ and $\alpha_{d}$ satisfying the given constraints.

The desired trajectory signal $q_{d}=\left[y_{d}, \alpha_{d}\right]^{T}$, which is continuous and bounded and differentiable up to 2 nd order. Let the tracking error $e=\left[e_{o}, e_{\alpha}\right]^{T}=$ $\left[y_{o}-y_{d}, \alpha-\alpha_{d}\right]^{T}$. Applied the output constraint, $e$ satisfies

$$
\begin{aligned}
& \left|e_{o}(t)\right|<k_{b 1}, \forall t \geq 0 \\
& \left|e_{\alpha}(t)\right|<k_{b 2}, \forall t \geq 0
\end{aligned}
$$

where $k_{b 1}=k_{c 1}-A_{01}, k_{b 2}=k_{c 2}-A_{02}$. Then, it can be seen, from (2), $\left|y_{d}\right| \leq A_{01}$ and $\left|\alpha_{d}\right| \leq A_{02}$, that $\left|y_{o}\right|<k_{b 1}+A_{01}=k_{c 1},|\alpha|<k_{b 2}+A_{02}=k_{c 2}$ and the constraint (8) can be kept.

The control objective of this paper is to design a synchronization control $u$ for uncertain leviation module (6) subjected to lumped mismatched disturbances $d(t)$ such that $y_{o}$ and $\alpha$ track $y_{d}$ and $\alpha_{d}$ as close as possibly, i.e., $y_{1}$ and $y_{2}$ is realized motion synchronization without module chattering. At the same time, $y_{o}$ and $\alpha$ can be stayed within the prescribed constrained regions during the operation. 


\section{DO based robust I\&I adaptive controller}

\subsection{The synchronization controller}

Firstly, define a switching-function-like quantity as

$$
s=\dot{e}+\Lambda e
$$

where $\Lambda=\operatorname{diag}\left[\Lambda_{11}, \Lambda_{22}\right]>0$. The transfer function matrix from $s$ to $e$ is $G(\mathbf{s})=E(\mathbf{s}) / S(\mathbf{s})=(I \mathbf{s}+\Lambda)^{-1}$, which implies that to make the quality $s$ small( or converges to zero) means $e$ small( or converges to zero) with the convergence rate determined by $\Lambda$. Note that $(6)$ and $(11)$, the derivative of $s$ can be written as

$$
\dot{s}=\theta_{2} v+\phi \theta_{1}+d-\ddot{q}_{d}+\Lambda \dot{e}
$$

To limit large and fast variations of $y_{0}$ and $\alpha$, define a Barrier Lyapunov Function as in 28

$$
V=\frac{1}{2} s^{T} s+\frac{1}{2} \ln \left(\frac{k_{b 1}^{2}}{k_{b 1}^{2}-e_{o}^{2}}\right)+\frac{1}{2} \ln \left(\frac{k_{b 2}^{2}}{k_{b 2}^{2}-e_{\alpha}^{2}}\right)
$$

where $\ln (\bullet)$ is the natural logarithm function of $\bullet$. The derivative of $V$ is calculated as follows,

$$
\begin{aligned}
\dot{V} & =s^{T}\left[\theta_{2} v+\phi \theta_{1}+d-\ddot{q}_{d}+\Lambda \dot{e}\right]+\frac{e_{y} \dot{e}_{o}}{k_{b 1}^{2}-e_{o}^{2}}+\frac{e_{\alpha} \dot{e}_{\alpha}}{k_{b 2}^{2}-e_{\alpha}^{2}} \\
& =s^{T}\left[\theta_{2} v+\phi \theta_{1}+d-\ddot{q}_{d}+\Lambda \dot{e}\right]+e^{T} K_{e} \dot{e}
\end{aligned}
$$

where $K_{e}=\operatorname{diag}\left[\frac{1}{k_{b 1}^{2}-e_{o}^{2}}, \frac{1}{k_{b 2}^{2}-e_{\alpha}^{2}}\right]>0$ since $\left|e_{o}(t)\right|<k_{b 1}$ and $\left|e_{\alpha}(t)\right|<k_{b 2}$.

If gain matrix $\theta_{2}^{-1}$ is known, one control law for 14 is designed as

$$
v=\theta_{2}^{-1} w
$$

where

$$
w=-K_{s} s-K_{e} e-\phi\left(\hat{\theta}_{1}+\beta_{1}(s)\right)-\hat{d}+\ddot{q}_{d}-\Lambda \dot{e}
$$

where $\theta_{2}^{-1}=\operatorname{diag}\left[\theta_{21}^{-1}, \theta_{22}^{-1}\right], w=\left[\begin{array}{ll}w_{1} & w_{2}\end{array}\right]^{T}, K_{s}>0$ is the gain matrix to be designed, $\hat{\theta}_{1}+\beta_{1}(s)$ is the estimate of $\theta_{1}$ to be recovered from I\&I adaptation 

designed disturbance observer later.

Since $\theta_{2}^{-1}$ is an unknown matrix, it is inconvenient for the I\&I adaptation law design and stability analysis. Noting that the form of $\theta_{2}^{-1}$, we introduce the following transformation with redefinition of the regressor matrix as

$$
\theta_{2}^{-1} w=\varphi \vartheta_{2}
$$

where $\vartheta_{2}=\left[\theta_{21}^{-1}, \theta_{22}^{-1}\right]^{T} \in \mathbb{R}^{2}$ and the regressor matrix $\varphi$

$$
\varphi=\left[\begin{array}{cc}
w_{1} & 0 \\
0 & w_{2}
\end{array}\right] \in \mathbb{R}^{2 \times 2} .
$$

Then, the control input 17 is rewritten as,

$$
v=\varphi\left(\hat{\vartheta}_{2}+\beta_{2}\left(s, \hat{\theta}_{1}, \hat{d}\right)\right)
$$

where $\hat{\vartheta}_{2}+\beta_{2}\left(s, \hat{\theta}_{1}, \hat{d}\right)$ is the estimate of $\vartheta_{2}$ with $\beta_{2}\left(s, \hat{\theta}_{1}, \hat{d}\right)=\left[\beta_{21}\left(s, \hat{\theta}_{1}, \hat{d}\right)\right.$, $\left.\beta_{22}\left(s, \hat{\theta}_{1}, \hat{d}\right)\right]^{T}$ to be designed in the following subsection.

Hence, according to (7), the control input $u$ is designed as

$$
\begin{aligned}
& u_{1}=i_{1}^{2}=\frac{\left(y_{o}+\alpha l\right)^{2}}{2}\left(v_{1}+\frac{1}{l} v_{2}\right) \\
& u_{2}=i_{2}^{2}=\frac{\left(y_{o}-\alpha l\right)^{2}}{2}\left(v_{1}-\frac{1}{l} v_{2}\right)
\end{aligned}
$$

\subsection{The I\&I adaptation law}

In this subsection, an adaptation law based on I\&I method is designed to estimate the unknown parameters in 6 .

Let us define the off-the-manifold coordinate

$$
\left\{\begin{array}{l}
z_{1}=\hat{\theta}_{1}+\beta_{1}(s)-\theta_{1} \\
z_{2}=\hat{\vartheta}_{2}+\beta_{2}\left(s, \hat{\theta}_{1}, \hat{d}\right)-\vartheta_{2}
\end{array}\right.
$$

where $\beta_{1}(s): \mathbb{R}^{2} \rightarrow \mathbb{R}^{5}$ and $\beta_{2}\left(s, \hat{\theta}_{1}, \hat{d}\right): \mathbb{R}^{2} \times \mathbb{R}^{5} \times \mathbb{R}^{2} \rightarrow \mathbb{R}^{2}$ are the tuning functions to be determined. 
Noting that 12), (17) and (20), Eq. 12 can be rewritten as

$$
\dot{s}=w+\theta_{2} \varphi z_{2}+\phi \theta_{1}+d-\ddot{q}_{d}+\Lambda \dot{e}
$$

The derivative of $z=\left[z_{1}, z_{2}\right]^{T}$ along the trajectories of (21) is calculated as

$$
\left\{\begin{aligned}
\dot{z}_{1}= & \dot{\hat{\theta}}_{1}+\frac{\partial \beta_{1}(s)}{\partial s}\left(w+\theta_{2} \varphi z_{2}+\phi \theta_{1}+d-\ddot{q}_{d}+\Lambda \dot{e}\right) \\
\dot{z}_{2}= & \dot{\hat{\vartheta}}_{2}+\frac{\partial \beta_{2}\left(s, \hat{\theta}_{1}, \hat{d}\right)}{\partial s}\left(w+\theta_{2} \varphi z_{2}+\phi \theta_{1}+d-\ddot{q}_{d}+\Lambda \dot{e}\right) \\
& +\frac{\partial \beta_{2}\left(s, \hat{\theta}_{1}, \hat{d}\right)}{\partial \hat{\theta}_{1}} \dot{\hat{\theta}}_{1}+\frac{\partial \beta_{2}\left(s, \hat{\theta}_{1}, \hat{d}\right)}{\partial \hat{d}} \dot{\hat{d}}
\end{aligned}\right.
$$

where

$$
\left\{\begin{array}{l}
\left(\frac{\partial \beta_{1}(s)}{\partial s}\right)_{i j}=\frac{\partial \beta_{1 j}}{\partial s_{i}}(s),(i, j) \in\{1,2\} \times\{1, \ldots, 5\} \\
\left(\frac{\partial \beta_{2}(s, \hat{\theta}, \hat{d})}{\partial s}\right)_{i k}=\frac{\partial \beta_{2 k}}{\partial s_{i}}\left(s, \hat{\theta}_{1}, \hat{d}\right),(i, k) \in\{1,2\} \times\{1,2\}
\end{array}\right.
$$

The I\&I adaptation laws of $\theta_{1}$ and $\vartheta_{2}$ are designed as

$$
\left\{\begin{aligned}
\dot{\hat{\theta}}_{1}= & -\frac{\partial \beta_{1}(s)}{\partial s}\left(w+\phi\left(\hat{\theta}_{1}+\beta_{1}(s)\right)+\hat{d}-\ddot{q}_{d}+\Lambda \dot{e}\right) \\
\dot{\hat{\vartheta}}_{2}= & -\frac{\partial \beta_{2}\left(s, \hat{\theta}_{1}, \hat{d}\right)}{\partial s}\left(w+\phi\left(\hat{\theta}_{1}+\beta_{1}(s)\right)+\hat{d}-\ddot{q}_{d}+\Lambda \dot{e}\right) \\
& -\frac{\partial \beta_{2}\left(s, \hat{\theta}_{1}, \hat{d}\right)}{\partial \hat{\theta}_{1}} \dot{\hat{\theta}}_{1}-\frac{\partial \beta_{2}\left(s, \hat{\theta}_{1}, \hat{d}\right)}{\partial \hat{d}} \dot{\hat{d}}
\end{aligned}\right.
$$

Lemma 1. If the function $\beta(\cdot)=\left[\beta_{1}(\cdot)^{T}, \beta_{2}(\cdot)^{T}\right]^{T}$ is selected such that

$$
\left\{\begin{array}{l}
\frac{\partial \beta_{1}(s)}{\partial s}=\Gamma_{1} \phi^{T} \\
\frac{\partial \beta_{2}\left(s, \hat{\theta}_{1}, \hat{d}\right)}{\partial s}=-\Gamma_{2} \varphi^{T}
\end{array}\right.
$$

where $\Gamma_{1}>0$ and $\Gamma_{2}>0$ are diagonal gain matrices, then the estimates $\hat{\Theta}+\beta(\cdot)$ can asymptotically converge its true values $\Theta$ with a bounded residual set related to $\tilde{d}$, in which $\hat{\Theta}=\left[\hat{\theta}_{1}^{T}, \hat{\vartheta}_{2}^{T}\right]^{T}$ and $\Theta=\left[\theta_{1}^{T}, \vartheta_{2}^{T}\right]^{T}$.

Proof: Substituting 23) and 24 into 22, yields the dynamics

$$
\left\{\begin{array}{l}
\dot{z}_{1}=-\Gamma_{1} \phi^{T} \phi z_{1}+\Gamma_{1} \phi^{T} \theta_{2} \varphi z_{2}+\Gamma_{1} \phi^{T} \tilde{d} \\
\dot{z}_{2}=-\Gamma_{2} \varphi^{T} \theta_{2} \varphi z_{2}+\Gamma_{2} \varphi^{T} \phi z_{1}-\Gamma_{2} \varphi^{T} \tilde{d}
\end{array}\right.
$$

Let $\Theta=\left[\theta_{1}^{T}, \vartheta_{2}^{T}\right]^{T}$, then $z=\hat{\Theta}+\beta(\cdot)-\Theta=\left[z_{1}^{T}, z_{2}^{T}\right]^{T}$. The dynamics 25 can rewritten as

$$
\dot{z}=-\Gamma_{z} \Phi\left(s, \hat{\theta}_{1}, \hat{d}\right) \Phi^{T}\left(s, \hat{\theta}_{1}, \hat{d}\right) z+\Gamma_{z} \Phi^{T}\left(s, \hat{\theta}_{1}, \hat{d}\right) \tilde{d}
$$


where $\Phi\left(s, \hat{\theta}_{1}, \hat{d}\right)=\left[\phi,-\theta_{2} \varphi\right]^{T}, \Gamma_{z}=\operatorname{diag}\left[\Gamma_{1}, \theta_{2}^{-1} \Gamma_{2}\right]$.

In order to show the convergence of $z$, define a positive-definite function $V_{z}=\frac{1}{2} z^{T} \Gamma \Gamma_{z}^{-1} z$, whose derivative along trajectory 26 is given

$$
\begin{aligned}
\dot{V}_{z} & =-z^{T} \Gamma \Phi \Phi^{T} z+z^{T} \Gamma \Phi \tilde{d} \\
& \leq-\gamma\left\|\Phi^{T} z\right\|^{2}+\frac{\gamma}{2}\left\|\Phi^{T} z\right\|^{2}+\frac{\gamma}{2}\|\tilde{d}\|^{2} \\
& =-\frac{\gamma}{2}\left\|\Phi^{T} z\right\|^{2}+\frac{\gamma}{2}\|\tilde{d}\|^{2}
\end{aligned}
$$

where $\gamma=\lambda_{\min }(\Gamma)$ is the minimal eigenvalue of $\Gamma$.

As a result, the boundedness of $z$ can be guaranteed, which implies that the estimate $\hat{\Theta}+\beta(\cdot)$ asymptotically converges to the neighbourhood of the true value $\Theta$ with an ultimate bound related to $\tilde{d}$.

\subsection{Disturbance observer design}

In this section, the following disturbance observer is proposed in control law to cancel disturbances $d(t)$ in (6),

$$
\left\{\begin{array}{l}
\dot{\xi}_{1}=-L_{1}\left(w+\phi\left(\hat{\theta}_{1}+\beta(s)\right)+\hat{d}\right)+\hat{\dot{d}} \\
\dot{\xi}_{2}=-L_{2}\left(w+\phi\left(\hat{\theta}_{1}+\beta(s)\right)+\hat{d}\right) \\
\hat{d}=\xi_{1}+L_{1} \dot{q} \\
\hat{\dot{d}}=\xi_{2}+L_{2} \dot{q}
\end{array}\right.
$$

where $\xi_{1}=\left[\xi_{11}, \xi_{12}\right]^{T}$ and $\xi_{2}=\left[\xi_{21}, \xi_{22}\right]^{T}$ are the state variables of the observer, $\hat{d}=\left[\hat{d}_{1}, \hat{d}_{2}\right]^{T}$ and $\hat{\dot{d}}=\left[\begin{array}{ll}\hat{\dot{d}}_{1}, & \hat{\dot{d}}_{2}\end{array}\right]^{T}$ are the estimate of $d=\left[d_{1}, d_{2}\right]^{T}$ and $\dot{d}=\left[\dot{d}_{1}, \dot{d}_{2}\right]^{T}$, respectively, $L_{1}=\operatorname{diag}\left[l_{11}, l_{12}\right]$ and $L_{2}=\operatorname{diag}\left[l_{21}, l_{22}\right]$ are positive observer gain matrices to be determined.

Define the disturbance observer errors $e_{d}=\left[\begin{array}{ll}\tilde{d}^{T} & \tilde{d}^{T}\end{array}\right]^{T}=\left[\begin{array}{lll}d^{T}-\hat{d}^{T} & \dot{d}^{T}-\hat{d}^{T}\end{array}\right]^{T}$. It can be derived from $(28)$ and $(6)$ that

$$
\begin{gathered}
\dot{\tilde{d}}=\dot{d}-\left(\dot{\xi}_{1}+L_{1} \ddot{q}\right)=-L_{1} \tilde{d}+\tilde{\dot{d}}+L_{1} \Phi^{T} z \\
\dot{\tilde{d}}=\ddot{d}-\left(\dot{\xi}_{2}+L_{2} \ddot{q}\right)=-L_{2} \tilde{d}+\ddot{d}+L_{2} \Phi^{T} z
\end{gathered}
$$

Then the dynamics of $e_{d}$ can be written as,

$$
\dot{e}_{d}=F e_{d}+D \ddot{d}+E \Phi^{T} z
$$


where

$$
F=\left[\begin{array}{cc}
-L_{1} & I_{2 \times 2} \\
-L_{2} & 0
\end{array}\right], D=\left[\begin{array}{cc}
0_{2 \times 2} & \\
& I_{2 \times 2}
\end{array}\right], E=\left[\begin{array}{c}
L_{1} \\
L_{2}
\end{array}\right]
$$

Lemma 2. If Assumption 1 holds, the disturbance estimate $\hat{d}$ and its first order derivative estimate $\hat{\dot{d}}$ generated by observer (28) can converge exponentially to $d$ and $\dot{d}$ with an ultimate bound related to $\ddot{d}$ if the gain matrices $L_{1}>0, L_{2}>0$ in 28) are chosen appropriately such that $F$ is Hurwitz.

Proof: Since $L_{1}>0$ and $L_{2}>0$ such that $F$ is Hurwitz matrix, there exists a matrix $P>0$ such that the following equation

$$
F P+P F^{T}=-Q_{1}
$$

holds for any given positive definite matrix $Q_{1}$.

Define a Lyapunov function

$$
V_{e_{d}}=e_{d}^{T} P e_{d}
$$

which satisfies the following inequalities

$$
\lambda_{\min }(P)\left\|e_{d}\right\|^{2} \leq V_{e_{d}} \leq \lambda_{\max }(P)\left\|e_{d}\right\|^{2}
$$

where $\lambda_{\min }(P)$ and $\lambda_{\max }(P)$ are the minimum and maximum eigenvalue of $P$, respectively.

The derivative of $V_{e_{d}}$ is calculated as

$$
\begin{aligned}
\dot{V}_{e_{d}} & =e_{d}^{T}\left(F P+P F^{T}\right) e_{d}+2 e_{d}^{T} P D \ddot{d}+2 e_{d}^{T} P E \Phi^{T} z \\
& \leq-\lambda_{\min }\left(Q_{1}\right)\left\|e_{d}\right\|^{2}+2\left\|e_{d}\right\| \lambda_{\max }(P)\left(\|D\| \mu+\|E\|\left\|\Phi^{T} z\right\|\right)
\end{aligned}
$$

where $\lambda_{\min }\left(Q_{1}\right)$ is the minimum eigenvalue of $Q_{1}$.

Since the Assumption 1 ensures the boundedness of the $\ddot{d}$ and $\Phi^{T} z$ is bounded from (27), we can conclude that the solution of the disturbance observer error dynamic 30 is uniformly ultimately bounded. 
Remark 4. From (35), we can show that the ultimate bound $b=2 \lambda_{\max }(P)$ $\frac{\left(\|D\| \mu+\|E\|\left\|\Phi^{T} z\right\|\right)}{\lambda_{\min }(Q)}$. The term $\left\|\Phi^{T} z\right\|$ can be decreased so that its effect is eventually dominated by choosing appropriately the gain matrix $\Gamma$ in (27). In addition, the bounds also can be lowered by increasing the observer gain matrices $L_{1}$ and $L_{2}$ and/or decreasing $\mu$.

\section{The main result and stability analysis}

In this section, the parameters of the control law will be determined and the stability analysis of the closed-loop leviation module is given.

To derive the main results, let us define the following Lyapunov function candidate as

$$
V_{a}=V+\frac{1}{2} z^{T} \Gamma \Gamma_{z}^{-1} z+\frac{1}{2} \tilde{d}^{T} \tilde{d}+\frac{1}{2} \tilde{\dot{d}}^{T} \tilde{\dot{d}} .
$$

Theorem 1. Consider the closed-loop system consisting of the system (6) satisfying Assumptions 1 to 2, the robust adaptive synchronization control law (19) with I\&I adaptation law (23) and disturbance observer (28). If the initial conditions $\left|e_{o}(0)\right|<k_{b 1}$ and $\left|e_{\alpha}(0)\right|<k_{b 2}$ are satisfied, there exist gain matrices $K_{s}, \Lambda, \Gamma$ and $L_{1}, L_{2}$, such that the following matrix is positive-definite

$$
\begin{aligned}
Q= & \operatorname{diag}\left[\lambda_{\min }\left(K_{s}\right)-\frac{\rho_{1}}{2}-\frac{\rho_{2}}{2}, \lambda_{\min }\left(K_{e} \Lambda\right), \gamma-\frac{1}{2 \rho_{1}}\right. \\
& -\frac{\rho_{3}\left(\gamma+\lambda_{\max }\left(L_{1}\right)\right)}{2}+\frac{\rho_{5} \lambda_{\max }\left(L_{2}\right)}{2}, \lambda_{\min }\left(L_{1}\right) \\
& -\frac{1}{2 \rho_{2}}-\frac{\left(\gamma+\lambda_{\min }\left(L_{1}\right)\right)}{2 \rho_{3}}+\frac{\rho_{4}\left(\lambda_{\min }\left(L_{2}\right)-1\right)}{2}, \\
& \left.\frac{\left(\lambda_{\min }\left(L_{2}\right)-1\right)}{2 \rho_{4}}-\frac{\lambda_{\max }\left(L_{2}\right)}{2 \rho_{5}}-\frac{\rho_{6}}{2}\right]
\end{aligned}
$$

where $\rho_{j}>0, j=1,2,3,4,5,6$ are positive constants, then the following properties hold.

1. The signals $s, e_{o}, e_{\alpha}$ remain in the following compact sets

$$
\begin{aligned}
\Omega_{s} & =\left\{s \in \mathbb{R}^{2}:\|s\| \leq \sqrt{2 V_{a}(0)}\right\} \\
\Omega_{e} & =\left\{e=\left[e_{o}, e_{\alpha}\right]^{T} \in \mathbb{R}^{2}: e_{o} \leq D_{e_{o}}, e_{\alpha} \leq D_{e_{\alpha}}\right\} \\
D_{e_{o}} & =k_{b 1} \sqrt{1-e^{-2 V_{a}(0)}}, D_{e_{\alpha}}=k_{b 2} \sqrt{1-e^{-2 V_{a}(0)}}
\end{aligned}
$$


where $V_{a}$ is the overall Lyapunov function candidate defined in (36).

2. The system output constraint requirements $\left|y_{o}(t)\right| \leq k_{c 1}, \forall t \geq 0$ and $|\alpha(t)| \leq k_{c 2}, \forall t \geq 0$ are satisfied, that is, the output constraints will not be violated during operation.

3. All the signals in the closed-loop system are ultimately bounded. Furthermore, the outputs will asymptotically converge to the domain around their true values with an ultimate bound $r$ related to $\ddot{d}$, that is

$$
\dot{V}_{a} \leq-x^{T} Q x+r
$$

where $x^{T}=\left[\|s\|,\|e\|,\left\|\Phi^{T} z\right\|,\|\tilde{d}\|,\|\tilde{d}\|\right]$.

Proof: Noting that (14), 27), 29), the derivative of $V_{a}$ is given as

$$
\begin{aligned}
\dot{V}_{a}= & -s^{T} K_{s} s-s^{T} \Phi^{T} z+s^{T} \tilde{d}-e^{T} K_{e} \Lambda e-z^{T} \Gamma \Phi \Phi^{T} z+z^{T} \Gamma \Phi \tilde{d} \\
& -\tilde{d}^{T} L_{1} \tilde{d}+\tilde{d}^{T} L_{1} \Phi^{T} z+\tilde{d}^{T} \tilde{\dot{d}}-\tilde{d}^{T} L_{2} \tilde{d}+\tilde{d}^{T} L_{2} \Phi^{T} z+\tilde{d}^{T} \ddot{d} \\
\leq & -\lambda_{\min }\left(K_{s}\right)\|s\|^{2}+\|s\|\left\|\Phi^{T} z\right\|+\|s\|\|\tilde{d}\| \\
& -\lambda_{\min }\left(K_{e} \Lambda\right)\|e\|^{2}-\gamma\left\|\Phi^{T} z\right\|^{2}-\lambda_{\min }\left(L_{1}\right)\|\tilde{d}\|^{2} \\
& +\left(\gamma+\lambda_{\max }\left(L_{1}\right)\right)\left\|\Phi^{T} z\right\|\|\tilde{d}\|-\left(\lambda_{\min }\left(L_{2}\right)-1\right)\|\tilde{d}\|\|\tilde{\dot{d}}\| \\
& +\lambda_{\max }\left(L_{2}\right)\left\|\Phi^{T} z\right\|\|\tilde{d}\|+\|\tilde{d}\|\|\ddot{d}\|
\end{aligned}
$$

where $\lambda_{\min }\left(K_{s}\right), \lambda_{\min }\left(K_{e} \Lambda\right), \lambda_{\min }\left(L_{1}\right)$ and $\lambda_{\min }\left(L_{2}\right)$ denote the minimum eigenvalue of gain matrices $K_{s}, K_{e} \Lambda, L_{1}$ and $L_{2}$, respectively. $\lambda_{\max }\left(L_{1}\right)$ and $\lambda_{\max }\left(L_{2}\right)$ are the maximum eigenvalue of $L_{1}$ and $L_{2}$, respectively.

Using Young's inequality to the cross-terms on the right-hand side of 40 
yields

$$
\begin{aligned}
\|s\|\left\|\Phi^{T} z\right\| & \leq \frac{\rho_{1}}{2}\|s\|^{2}+\frac{1}{2 \rho_{1}}\left\|\Phi^{T} z\right\|^{2}, \\
\|s\|\|\tilde{d}\| & \leq \frac{\rho_{2}}{2}\|s\|^{2}+\frac{1}{2 \rho_{2}}\|\tilde{d}\|^{2}, \\
\left\|\Phi^{T} z\right\|\|\tilde{d}\| & \leq \frac{\rho_{3}}{2}\left\|\Phi^{T} z\right\|^{2}+\frac{1}{2 \rho_{3}}\|\tilde{d}\|^{2}, \\
\|\tilde{d}\|\|\tilde{\dot{d}}\| & \leq \frac{\rho_{4}}{2}\|\tilde{d}\|^{2}+\frac{1}{2 \rho_{4}}\|\tilde{d}\|^{2}, \\
\left\|\Phi^{T} z\right\|\|\tilde{d}\| & \leq \frac{\rho_{5}}{2}\left\|\Phi^{T} z\right\|^{2}+\frac{1}{2 \rho_{5}}\|\tilde{\dot{d}}\|^{2}, \\
\|\tilde{\dot{d}}\|\|\tilde{d}\| & \leq \frac{\rho_{6}}{2}\|\tilde{\dot{d}}\|^{2}+\frac{1}{2 \rho_{6}}\|\tilde{d}\|^{2}
\end{aligned}
$$

Substituting (41) into (40), we have

$$
\begin{aligned}
\dot{V}_{a} \leq & -\left(\lambda_{\min }\left(K_{s}\right)-\frac{\rho_{1}}{2}-\frac{\rho_{2}}{2}\right)\|s\|^{2}-\lambda_{\min }\left(K_{e} \Lambda\right)\|e\|^{2} \\
& -\left(\gamma-\frac{1}{2 \rho_{1}}-\frac{\rho_{3}\left(\gamma+\lambda_{\max }\left(L_{1}\right)\right)}{2}+\frac{\rho_{5} \lambda_{\max }\left(L_{2}\right)}{2}\right)\left\|\Phi^{T} z\right\|^{2} \\
& -\left(\lambda_{\min }\left(L_{1}\right)-\frac{\left(\gamma+\lambda_{\min }\left(L_{1}\right)\right)}{2 \rho_{3}}+\frac{\rho_{4}\left(\lambda_{\min }\left(L_{2}\right)-1\right)}{2}\right. \\
& \left.-\frac{1}{2 \rho_{2}}\right)\|\tilde{d}\|^{2}-\left(\frac{\left(\lambda_{\min }\left(L_{2}\right)-1\right)}{2 \rho_{4}}-\frac{\lambda_{\max }\left(L_{2}\right)}{2 \rho_{5}}-\frac{\rho_{6}}{2}\right)\|\tilde{d}\|^{2}+\frac{1}{2 \rho_{6}}\|\ddot{d}\|^{2}
\end{aligned}
$$

Note that (37) and the definition of $x$ in $(39)$, Eq. 42 can be re-expressed as

$$
\dot{V}_{a} \leq-x^{T} Q x+r
$$

where $r=\frac{1}{2 \rho_{6}}\|\ddot{d}\|^{2}$.

(1) From (43), it follows that $V_{a}(t) \leq V_{a}(0)$. Thus, we can obtain

$$
\frac{1}{2} \ln \left(\frac{k_{b 1}^{2}}{k_{b 1}^{2}-e_{o}^{2}(t)}\right) \leq V_{a}(0), \frac{1}{2} \ln \left(\frac{k_{b 2}^{2}}{k_{b 2}^{2}-e_{\alpha}^{2}(t)}\right) \leq V_{a}(0)
$$

Then from (44), we have

$$
\left|e_{o}(t)\right| \leq k_{b 1} \sqrt{1-e^{-2 V_{a}(0)}},\left|e_{\alpha}(t)\right| \leq k_{b 2} \sqrt{1-e^{-2 V_{a}(0)}}
$$

Similarly, due to the fact that $\frac{1}{2} s^{T} s \leq V_{a}(0)$, we can get the $\|s\| \leq \sqrt{2 V_{a}(0)}$ as in the theorem statement.

(2) From (1), we know that $\left|e_{o}(t)\right| \leq D_{e_{o}}<k_{b 1}$ and $\left|e_{\alpha}(t)\right| \leq D_{e_{\alpha}}<k_{b 2}$ can be satisfied. Since $y_{o}=e_{o}+y_{d}, \alpha=e_{\alpha}+\alpha_{d},\left|y_{d}\right| \leq A_{01}$ and $\left|\alpha_{d}\right| \leq A_{02}$, we 
can conclude that $\left|y_{o}\right|<k_{b 1}+A_{01}=k_{c 1}$ and $|\alpha|<k_{b 2}+A_{02}=k_{c 2}$. That is, the

(3) From (43), it is can be seen that the signals $s, e, z, \tilde{d}, \tilde{\dot{d}}$ are bounded from function $r$. Together with the fact that $d$ is bounded up to its second derivatives at least from Assumption 1, it is clear that $\hat{d}$ and $\hat{\dot{d}}$ are bounded. Since the $\hat{\Theta}+\beta(\cdot)$ is bounded and the given desired trajectory signals are bounded up to 290 guaranteed. Therefore, all the signals in the closed-loop system are uniformly bounded. Finally, from (43), it implies that the output signals asymptotically converge to the domain around their true values with an ultimate bound related to $\ddot{d}$ and $\ddot{d}$ satisfies the Assumption 1 .

${ }_{295}$ Remark 5. The design parameters $K_{s}, \Lambda, \Gamma, L_{1}, L_{2}$ and $\rho_{j}, j=1,2,3,4,5,6>$ 0 should synthetically selected to make matrix $Q$ positive-definite such that satisfying tracking performance is obtained.

Remark 6. Actually, the desired value of current $i$ is computed by

$$
i_{1}=u_{1}^{0.5}, i_{2}=u_{2}^{0.5}
$$

it is required here $u_{1} \geq 0, u_{2} \geq 0$. These inequalities are satisfied in generic cases if design parameters $K_{s}, \Lambda, \Gamma, L_{1}, L_{2}$ and $\rho_{j}, j=1,2,3,4,5,6>0$ are chosen to be extremely large and the bound values $k_{b 1}, k_{b 2}$ are not chosen to be extremely small.

\section{Numerical simulation}

To verify the effectiveness of the proposed robust I\&I adaptive synchronization controller for an output-constrained uncertain maglev system subjected to mismatched disturbances, a series of numerical simulations are carried out in this section.

Considering an actual requirement of leviation module as an example. The objective of this paper is to make $y_{o}$ track desired trajectory $y_{d}=0.009+$ 
$0.001 \sin (0.05 \pi t) m$ and the rotational angle $\alpha$ track $\alpha_{d}=0$, the output is subject to the constraint $\left|y_{o}(t)\right| \leq k_{c 1}=0.013 m, \forall t \geq 0$, meanwhile, $|\alpha(t)| \leq$ $k_{c 2}=0.002 \mathrm{rad}, \forall t \geq 0$. Since $0.008 \leq y_{d} \leq 0.01$ and $\alpha_{d}=0$, we obtain that $k_{b 1}=0.013-0.01=0.003$ and $k_{b 2}=0.002$. The nominal values of the parameters of levitation module are taken as $m=800 \mathrm{~kg}, J=850 \mathrm{kgm}^{2}$, $g=9.8 \mathrm{~m} / \mathrm{s}^{2}, N_{01}=10000 \mathrm{~N}, N_{02}=12000 \mathrm{~N}, K=0.0015 \mathrm{Nm}^{2} / \mathrm{A} . l=0.66 \mathrm{~m}$.

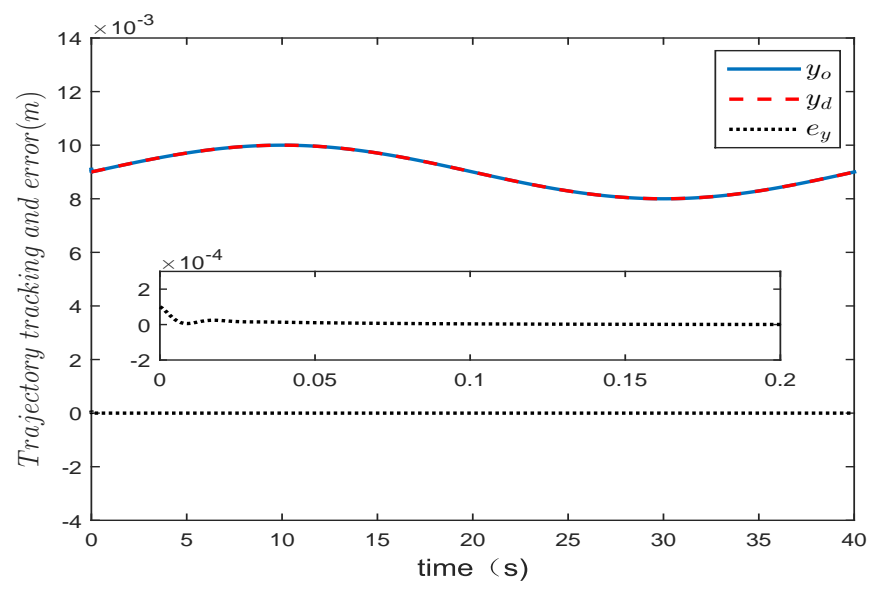

Figure 2: The curves of desired trajectory and actual trajectory.

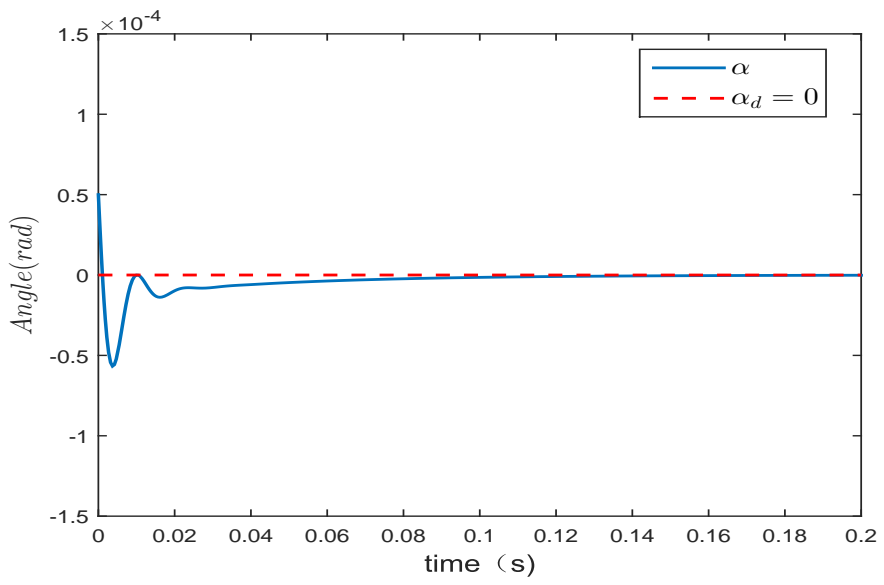

Figure 3: The curves of angle tracking. 
The mismatched disturbances are $d_{y}=100+100 \sin (0.1 \pi t), d_{\alpha}=10+$ $10 \cos (0.8 \pi t)$ and the small perturbations of load forces $\Delta N_{1}=200+100 \sin (0.2 \pi t) N$, $\Delta N_{2}=200+100 \cos (0.2 \pi t) N$, whose second derivatives satisfy Assumption 1. The initial conditions of the maglev system are $y_{o}(0)=0.0091 \mathrm{~m}$ and $\alpha(0)=0.00005 \mathrm{rad}$. Meanwhile, the initial parameter estimates are taken as $\hat{\Theta}(0)=\left[11,13,9.5,13.5,12,-5.4 \times 10^{5},-5.7 \times 10^{5}\right]^{T}$. The controller parameters are chosen as $K_{s}=\operatorname{diag}[150,200], \Lambda=\operatorname{diag}[150,200]$. The adaptation gain matrix are $\Gamma=\operatorname{diag}[8,10,2,5,8,1000,2000]$. The disturbance observer gain matrices are chosen as $L_{1}=\operatorname{diag}[20,30]^{T}$ and $L_{2}=\operatorname{diag}[10,20]^{T}$.

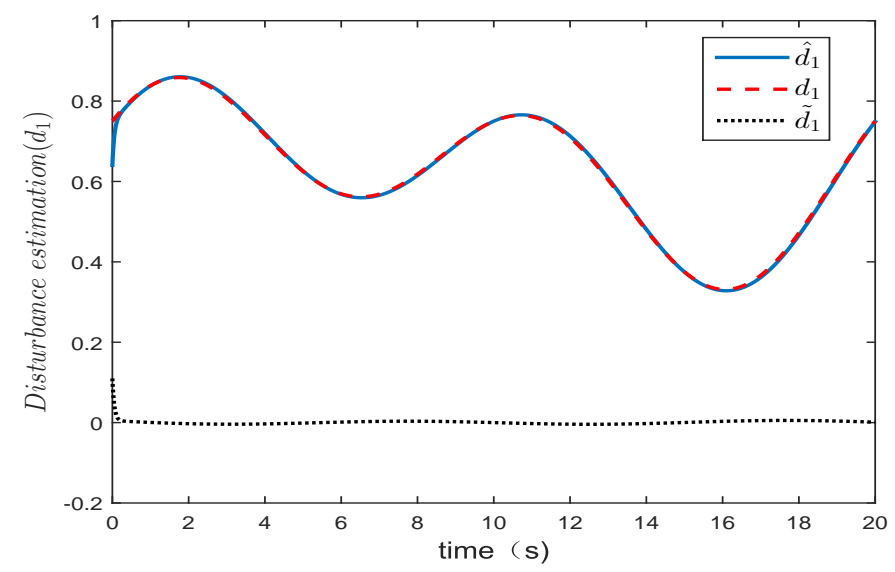

Figure 4: The curves of actual disturbance, estimated disturbance and estimated disturbance error $\left(d_{1}\right)$.

The simulation results are shown from Fig 2 to Fig 8 In Fig, 2 , it can be seen that the output $y_{o}$ can track the desired signal $y_{d}$ and always stay strictly within the given constraint $\left|y_{o}(t)\right| \leq k_{c 1}=0.013 m, \forall t \geq 0$. As can be seen in Fig 2 , the ordinate range of the trajectory tracking error is given as is $[-0.0002 m, 0.0002 m]$, which shows the trajectory tracking error constraint $\left|e_{o}(t)\right|<0.0002<k_{b 1}=0.003 \mathrm{~m}$. Meanwhile, Fig 3 gives the ordinate range as ${ }_{330}\left[-1.5 \times 10^{-4} \mathrm{rad}, 1.5 \times 10^{-4} \mathrm{rad}\right]$, it shows that rotational angle $\alpha$ converges to zero and varies within the set $|\alpha(t)| \leq 1.5 \times 10^{-4} \leq k_{c 2}=0.002 \mathrm{rad}, \forall t \geq 0$ during operation. The states of disturbance observer and their estimation errors are 


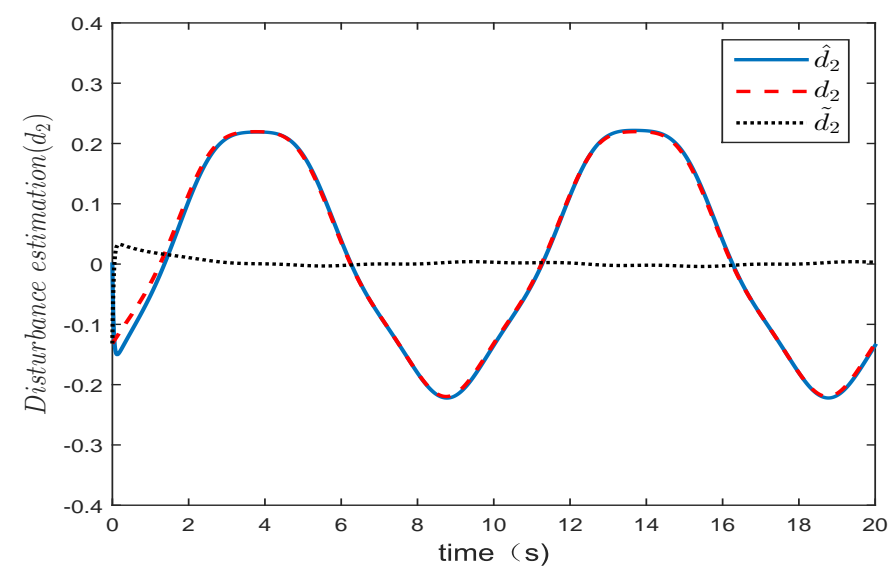

Figure 5: The curves of actual disturbance, estimated disturbance and estimated disturbance error $\left(d_{2}\right)$.
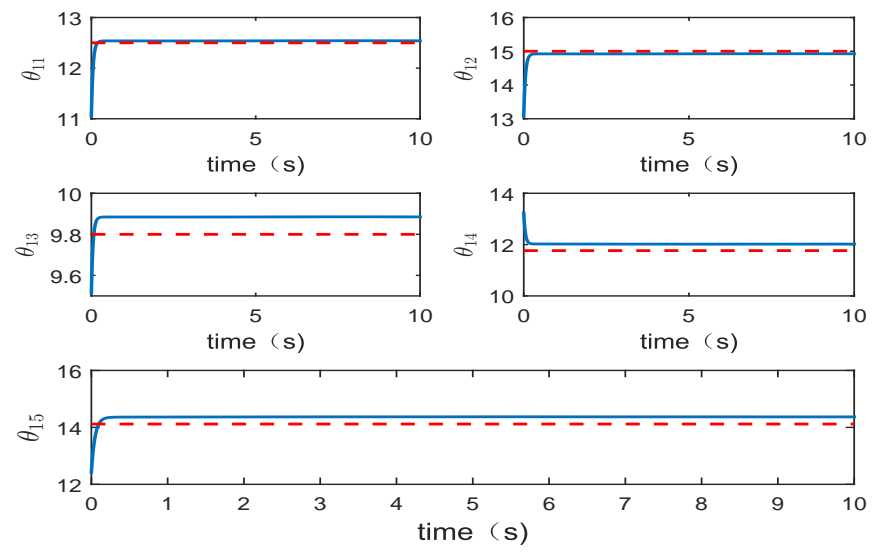

Figure 6: The curves of actual parameters and its estimated parameters $\left(\theta_{1}\right)$.

plotted in Fig 4 and Fig 5 , it can be seen that the disturbance observer cannot recover the disturbance completely since $\ddot{d}$ cannot be learned. The parameter estimates are plotted in Fig 6 and Fig 7 , where the dashed lines are the actual parameters and solid lines are the estimated parameters, which imply that parameter estimates can converge to their desired value. The control inputs $i_{1}, i_{2}$ are given in Fig,8. In a word, the effectiveness and superiority of the proposed 

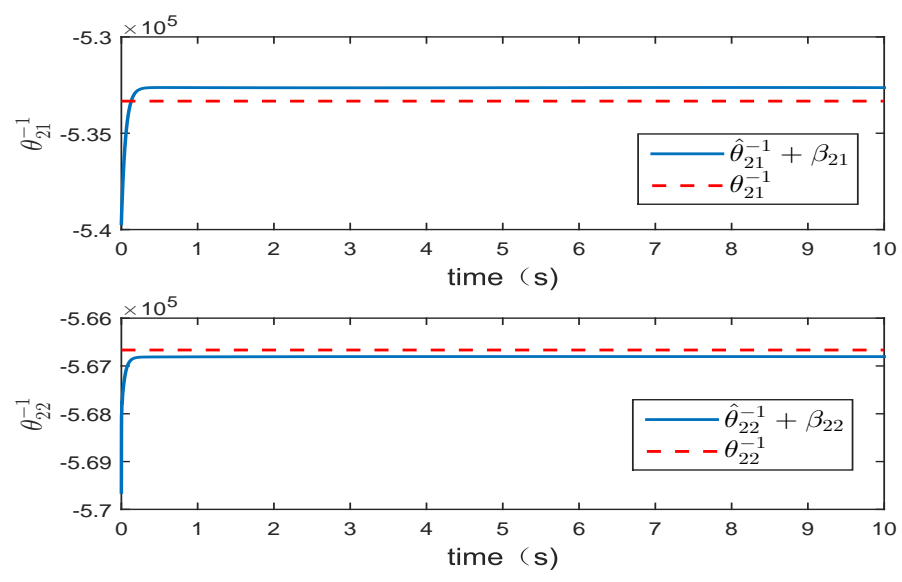

Figure 7: The curves of actual parameters and its estimated parameters $\left(\theta_{2}^{-1}\right)$.
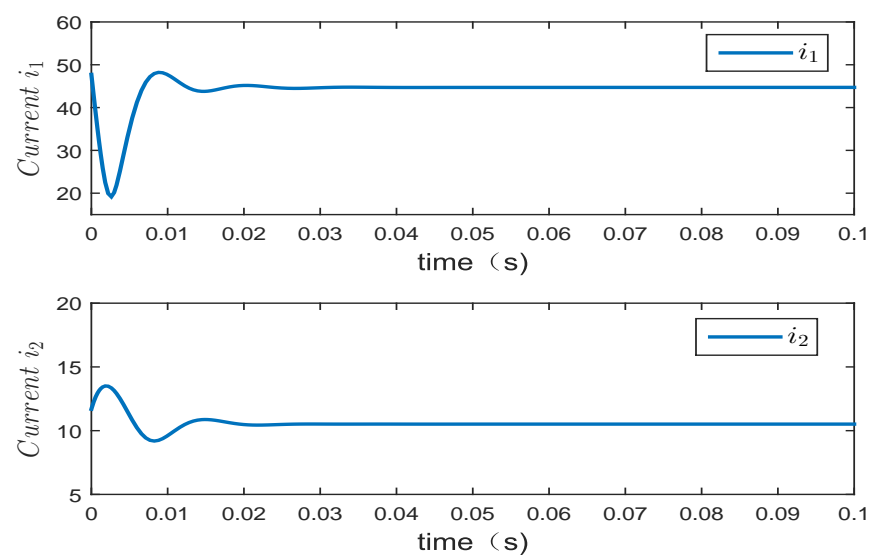

Figure 8: The curves of currents.

control law to achieve perfect tracking of $y_{o}$ and high level synchronization of two levitation units with force disturbances have been verified.

\section{Conclusions}

In this paper, a robust I\&I adaptive synchronization controller with DO is proposed for output-constrained uncertain maglev module subjected to mismatched disturbance. Firstly, with full consideration of mechanical coupling 
el was built to the maglev module, including vertical linear motion and the rotational motion around the mass center, implying clearly the interrelation of the mechanical coupling between the two maglev units. The Barrier Lyapunov Function is introduced into controller design to guarantee the outputs satisfy bance of maglev systems for desired operation performance, a robust adaptation law based on I\&I approach is developed to recover the unknown parameters in system. Simultaneously, an extended disturbance observer is constructed in the controller to alleviate the bad impact of the mismatched disturbance on the conobserver gains are chosen systematically such that the overall leviation module is stable with desired synchronization performance. The stability analysis shows that all the signals in the closed-loop system are ultimately bounded and output constraints are not violated as well. At last, the numerical simulation results verify the effectiveness of the proposed synchronization controller.

Conflict of Interest: The authors declare that they have no conflict of interest.

365 Funding: This study was funded by National Natural Science Foundation of China, Grant/Award Number: 61673050.

Data availability statement: All data generated or analysed during this study are included in this published article (and its supplementary information files). The datasets generated during the current study are available from the corresponding author on reasonable request.

Jiaqi Liu received her BS degree from Xiangtan University, China, in 2017, and MS degree from Beijing Jiaotong University, China, in 2020. Her interests includes adaptive control, nonlinear control and maglev train control.

375 Xiangbin Liu received his BS degree from Xian Institute of Technology, China, 
in 1995, MS degree from University of Science and Technology Beijing, China, in 2002, and Ph.D. degree from Zhejiang University, China, in 2009, respectively. He is an associate professor at the School of Electronics and Information Engineering, Beijing Jiaotong University. His research interests include adaptive control, robust control and nonlinear control.

Min Zhang received her BS degree from Zhengzhou University, China, in 2019. She is currently working toward the Ph.D. degree in the School of Electronic Information Engineering, Beijing Jiaotong University, Beijing, China. Her current research interests include adaptive control, nonlinear control and Fault-tolerant Control.

\section{References}

[1] H. W. Lee, K. C. Kim, and J. Lee, Review of maglev train technologies, IEEE Transactions on Magnetics, 2006, 42(7): 1917 - 1925. doi:10.1109/tmag.2006.875842.

[2] A. R. Eastham and W. F. Hayes, Maglev systems development status, IEEE Aerospace and Electronics Systems Magazine, 1988, 3(1): 21C30. doi:10.1109/62.843.

[3] L. G. Yan, Development and application of the maglev transportation system. IEEE Transactions on Applied Superconductivity, 2008, 18(2): 92 -99. doi:10.1109/tasc.2008.922239.

[4] R. Meisinger, Simulation of maglev vehicles riding over single and double span guideways, Mathematics and Computers in Simulation, 1979, 21(2): 197-206. doi:10.1109/tmag.2006.875842.

[5] Y. Cai, S. S. Chen, D. M. Rote and H. T. Coffey, Vehicle/guideway interaction for high speed vehicles on a flexible guideway, 1994, 175(5): 625C646. doi:10.1006/jsvi.1994.1350. 
[6] H.S. Han, B.H. Yim, N.J. Lee, et al, Effects of the guideway's vibrational characteristics on the dynamics of a maglev vehicle, Vehicle System Dynamics, 2009, 47(3): 309C324. doi:10.1080/00423110802054342.

[7] D.F. Zhou, P.C. Yu, L.C. Wang, et al, An adaptive vibration control method to suppress the vibration of the maglev train caused by track irregularities. Journal of Sound and Vibration, 2017: 331-350. doi:0.1016/j.jsv.2017.07.037.

[8] P.C. Yu, J.Li, L.C. Wang, Research on the influence of track periodic short-wave irregularity on low-speed maglev train, in: Proceedings of the 35th Chinese Control Conference, 2016: 9305-9310. doi:10.1109/ChiCC.2016.7554836.

[9] C. Li, Cong. Li, Z.Chen, et al, Advanced synchronization control of a dual-linear-motor-driven gantry with rotational dynamics, IEEE Transactions on Industrial Electronics, 2018, 64(9): 7526-7535. doi:10.1109/TIE.2018.2795522.

[10] Cong. Li, B. Yao, Q.F. Wang, Modeling and synchronization control of a dual drive industrial gantry stage, IEEE/ASME Transactions on Mechatronics, 2018, 23(6):2940-2951. doi:10.1109/TMECH.2018.2874876.

[11] K.P. Tee, S.S. Ge, and E.H. Tay, Barrier Lyapunov Functions for the control of output-constrained nonlinear systems, Automatica, 2009, 45(4): 918927. doi:10.1016/j.automatica.2008.11.017.

[12] X. Jin, Adaptive fixed-time control for MIMO nonlinear systems with asymmetric output constraints using universal barrier functions, IEEE Transactions on Automatic Control, 2018, 64(7): 3046-3053. doi:10.1109/TAC.2018.2874877.

[13] C. MacLeod, R. M. Goodall. Frequency-shaping LQ control of Maglev suspension systems for optimal performance with deterministic and stochastic 
inputs. IEEE Proceedings-Control Theory and Applications, 1996, 143(1): 25-30. doi:10.1049/ip-cta:19960057 .

[14] K. Michail, A. C. Goodall, R. M, Whidborne, et al, Optimised configuration of sensing elements for control and fault tolerance applied to an elctro-magnetic suspension system. International Journal of Systems Science, 2011, 43(10): 1785-1804. doi:10.1080/00207721.2011.598959.

[15] J. Yang, W. H. Chen, S. Li, Nonlinear disturbance-observer based robust control for systems with mismatched disturbances/uncertainties, IET Control Theory and Applications, 2011, 5(18): 2053C2062. doi: 10.1049/ietcta.2010.0616.

[16] J. Yang, S. Li, W. H. Chen, Nonlinear disturbance observer-based control for multi-input multi-output nonlinear systems subject to mismatching condition, International Journal of Control, 2012, 85(8): 1071C1082. doi: 10.1080/00207179.2012.675520.

[17] J. Yang, A. Zolotas, W. H. Chen, et al, Robust control of nonlinear MAGLEV suspension system with mismatched uncertainties via DOBC approach, ISA Transactions, 2011, 50(3): 389 C396. doi:10.1016/j.isatra.2011.01.006.

[18] J. Yang, S. Li, X. Yu, Sliding mode control for systems with mismatched uncertainties via a disturbance observer, IEEE Transactions on Industrial Electronics, 2013, 60(1): 160-169. doi:10.1109/tie.2012.2183841.

[19] J. Yang, A. Zolotas, and W. H. Chen, Robust control of nonlinear MAGLEV suspension system with mismatched uncertainties via DOBC approach, ISA Transactions, 2011, 50(3): 389-396. doi:10.1016/j.isatra.2011.01.006.

[20] D. Ginoya, P. D. Shendge, and S. B. Phadke, Sliding mode control 455 for mismatched uncertain systems using an extended disturbance observ- 
er, IEEE Transactions on Automatica Control, 2013, 61(4): 1983-1992. doi:10.1109/tie.2013.2271597 .

[21] Z. Z. Zhang, X. L. Li. Real-time adaptive control of a magnetic levitation system with a large range of load disturbance. Sensors, 2018, 18(5): 15121527. doi:10.3390/s18051512.

[22] Y. Zhang; Akujuobi, C. M. Akujuobi, W. H. Ali, et al, Load Disturbance Resistance Speed Controller Design for PMSM. IEEE Transactions on Industrial Electronics, 2006, 53(4): 1198-1208. doi:10.1109/tie.2006.878313.

[23] S. Lei, L. H. She, Research on rejecting load disturbance for levitation system of maglev train. In Proceedings of the Fourth International Symposium on Computational Intelligence and Design, 2011: 306C309. doi:10.1109/ISCID.2011.84.

[24] B. D. O. Anderson, Failures of adaptive control theory and their resolution[J]. Communications in Information and Systems, 2005, 5(1):1-20. doi: 10.4310/CIS.2005.v5.n1.a1.

[25] A. Astolfi, and R. Ortega, Immersion and Invariance: a new tool for stabilization and adaptive control of nonlinear systems, IEEE Transactions on Automatic control, 2003, 48(4): 590-606. doi:10.1109/TAC.2003.809820.

[26] M. Krstic, I. Kanellakopoulos, and P. Kokotovic, Nonlinear and adaptive control design, A Wiley-Interscience Publication, 1995.

[27] X. B. Liu, R. Ortega, H. Y. Su, et al, Immersion and invariance adaptive control of nonlinearly parameterized nonlinear systems, IEEE Transactions on Automatic Control, 2010, 55(9): 2209-2214. doi:10.3182/20100901-3-it2016.00294 .

${ }_{480}[28]$ K. B. Ngo, R. Mahony, Z. P. Jiang, Integrator backstepping using Barrier Functions for systems with multiple state constraints. Decision and Control, 2005 and 2005 European Control Conference. CDC-ECC '05. 44th IEEE Conference on IEEE, 2006. doi:10.1109/CDC.2005.1583507. 